\title{
Incredible Years Parent Training: Does it Improve Positive Relationships in Portuguese Families of Preschoolers with Oppositional/Defiant Symptoms?
}

\author{
Tatiana Carvalho Homem, Maria Filomena Gaspar, Maria João Seabra-Santos, Andreia \\ Azevedo \& Maria Cristina Canavarro
}

\begin{abstract}
Externalizing behavior disorders in children are difficult to cope with and might have a negative impact both on parent-child interactions and couples relationships, as well as on parents' psychological adjustment. The present study evaluates the effectiveness of a parent training program (Incredible Years Basic Program) for improving positive relationships in Portuguese families of preschoolers showing symptoms of oppositional/defiant disorder (ODD). Eighty three families randomly assigned to an Incredible Years (IY) intervention ( $n=44)$ or to a waiting list control group ( $\mathrm{n}=39$ ) were evaluated at baseline and six months after (post-intervention), on the following variables: mothers observed positive parenting practices and coaching skills; mothers' self- reported dysfunctional parenting practices; mothers' depressive symptoms; perceived couples intimacy; and children's behavior. Moreover, families who had received the IY program were also assessed in the same variables 12-months after baseline. Observed and self-reported positive parenting practices, couples openness to exterior and children's oppositional behavior improved more significantly from pre- to post-assessment in the intervention group than in the control group (medium to large effect sizes). Changes observed in the intervention group were sustained at the 12-month follow-up. However, the improvements in coaching skills previously observed at the post-intervention assessment in the intervention group decreased over time. The findings indicate that the IY Parent Program is a promising intervention to promote more positive parent-child relationships, decrease children's oppositional behaviors and develop couple's willingness to open to the exterior, from the mother's point of view.
\end{abstract}

Key-Words: Incredible Years; family positive relationships; ODD symptoms; preschoolers

\section{Introduction}

Oppositional/Defiant Disorder (ODD) is characterized by a recurrent pattern of negativistic, defiant, disobedient, and hostile behavior toward authoritary figures (APA, 2000). 
Along with Attention Deficit/Hyperactivity Disorder (ADHD), it is one of the major problems for which parents seek help from child mental health services. Despite the lack of consensus as to whether or not young children should be diagnosed with ODD (Wakschlag et. al., 2007), it is becoming clear to the clinicians that nearly half of the preschoolers whose parents seek mental health services for disruptive behavior go on exhibiting behavior problems in school and adolescent years (Campbell, Shaw, \& Gilliom, 2000; Rockhill, Collett, McClellan, \& Speltz, 2006). In addition, treatment outcome studies suggest that interventions for these disorders are of limited effect when offered in adolescence (Webster-Stratton, Gaspar, \& Seabra-Santos, 2012), highlighting the need for early intervention with these children and their families. In Portugal, there are no studies about ODD prevalence rates in preschool or school ages. However, some studies (Rijo, Motta, Silva, \& Brazão, 2013) show that $77.40 \%$ of Portuguese juvenile delinquents suffer from disruptive behavioral disorder. These results reinforce the importance of early intervention in children mental health. Moreover, behavior problems in children cause considerable economic costs to the community, but the burden still falls more heavily on the family (Fossum, Handegard, Martinussen, \& Morch, 2008). The present study evaluates the efficacy of the Incredible Years Basic Parent Training (IY) in improving the quality of motherchild relationship, mothers psychological functioning, marital quality and children's behavior in a sample of Portuguese families with preschoolers presenting early symptoms of ODD.

Developmental models of disruptive behavior problems have identified several risk factors from a number of different areas contributing to child behavior problems and it is now generally agreed that there are multiple pathways leading to these problems. Thus, an ecological and developmental model is needed in order to understand the development and maintenance of early behavior problems in children. In fact, along with the child's characteristics (e.g, temperament, neurophysiological and cognitive functions), environmental factors (such as parents' characteristics and parent-child attachment) can contribute to the emergence of behavior problems in some children, while in others they serve to escalate or stabilize them (Blommquist \& Schnell, 2002). Interactional parenting models suggest that elements of children's environments (e.g., parenting practices, family functioning) influence children's behavior and development in different ways depending on children's characteristics (Patterson \& Sanson, 1999). Patterson's anti-social behaviour developmental model (2002) emphasizes childrearing or the parent-child relationship qualities as central to the emergence and maintenance of children behaviour problems. Also, from a transactional and ecological perspective (Sameroff, 2009), early parent-child conflict may set the stage for more prolonged coercive exchanges that become characteristic of the parent-child relationship. Finally, Belsky's ecological model (1984) proposes that parenting practices are multiply determined by the interaction of parent's personal 
resources, children's characteristics and social contextual factors, such as marital relations. These factors interact with each other to influence parenting function which in turn influences children behaviour.

Even if it isn't the only influence on children's behavior, parenting has an important role in the development and maintenance of oppositional and aggressive behavior in childhood. As highlighted by Patterson, DeGarmo and Forgatch (2004), ineffective parenting skills, coercive and punitive discipline methods, and lack of monitoring are key factors contributing to child behavioral problems during preschool and elementary school years. Low levels of positive parenting behaviors, such as maternal responsiveness (Shaw, Keenan, \& Vondra, 1994) and positive involvement (Gardner, 1994) have also been implicated in the development and maintenance of early behavior problems in children as the stressful and demanding nature of the child's ODD characteristics are likely to evoke negative reactions from other family members and to have a strong impact on family relationships and on the psychological functioning of the parents (for a review see Campbell, 1995). Moreover, parent-child interaction difficulties in families of children with behavior problems have been observed and studies have found that mothers these children' mothers are more directive and negative and less rewarding, as well as more rejecting and aggressive (e.g., Gomez \& Sanson, 1994; Shaw, Owens, Giovannelli, \& Winslow, 2001). Also, higher behavior problem scores are associated with lower levels of family functioning, and parents of children with higher behavior problems levels report not having much time for themselves as well as higher levels of parental worry, when compared with parents of children with lower levels of problems (Sawyer et al., 2005). Additionally, studies have shown that parents of oppositional/defiant children are distressed in their parenting roles by their children oppositional and aggressive behaviors (Podolski \& Nigg, 2001). Baker and Heller (1996), for example, described the experiences of parents as a function of severity of child disruptive behavior in a community sample of preschool children. Their results suggest that parents of children with moderate and high levels of disruptive behavior experienced more child-related stress, a lower sense of parenting efficacy and were more authoritarian and indifferent in their parenting than parents of non-disruptive children. Finally, the link between couple relationship satisfaction and distress, parenting, and child outcomes is well established (Linville et al., 2010) and research has shown that marital problems are associated with angry and non-compliant children (Katz \& Gottman, 1994).

In conclusion, parent functioning, parent-child relationships and couples interactions are strongly related to child development, both as risk and as protective factors. Therefore, effective interventions must target these variables in order to prevent behavior problems in children (Cummings et al., 2000). Coping with troubled children is difficult for parents, as they require 
close supervision and control. In this context, many behavioral parent programs (BPP) have been developed for helping parents to deal with their children behavior problems (e.g. Sanders, MarkieDadds, Tully, \& Bor, 2000 [Triple P]; Matos, Bauermeister, \& Bernal, 2009 [Parent-Child Interaction Therapy]).

In Portugal there is a growing awareness of the potential of parenting programs as promotion, prevention and intervention strategies to improve children and families well-being (Gaspar \& Seabra-Santos, 2008). However, evidence-based parenting interventions (EBPI) have not attracted much interest or recognition in our country and few studies have been done on such interventions. The parenting program selected for the present study is the Incredible Years Basic Parent Training (IY; Webster-Stratton, 2001), a manualised program identified as a Blueprints Promising Program by the Center for Violence Prevention at the University of Colorado (http://www.colorado.edu/cspv/blueprints/modelprograms.html) and recommended by the American Psychological Association Task Force as meeting criteria for empirically supported mental health intervention for children (3-8 years old) with conduct problems (http://www.incredibleyears.com). The IY is one of the most researched and empirically supported psychosocial interventions for children (3-8 years old) with behavior problems and is used and studied internationally as a treatment for children with severe conduct problems, as well as a preventive intervention (Menting, Orobio de Castro and Matthys, 2013). Its efficacy and effectiveness for children with oppositional/defiant disorder has been evaluated in a series of international randomized control studies (Webster-Stratton, 2011) and in a number of successful replications (Bywater et al., 2009; Hutchings et al., 2007). Recently, in a randomized controlled trial (RCT) using a 14-week IY intervention program with parents of 103 children with behavioral problems, results showed that after the IY program there is a significant improvement both in behavior problems among children and in families well-being, as well as in parenting competencies compared to a wait-list control group (McGilloway et al., 2012). Also, another RCT using a 10-week IY intervention program with parents of 212 children who acknowledged disruptive behaviors on a 20 -item checklist, showed that compared to the wait list group, greater improvement was observed in the intervention group on what concerns parenting practices and child disruptive behaviors (Perrin, Sheldrick, McMenamy, Henson, \& Carter, 2013). Moreover, a recent meta-analysis on the effectiveness of the Incredible Years Parent Training regarding disruptive behavior, which included fifty studies (where an IY intervention group was compared to a control group) (Menting et al., 2013) showed that positive effects were found for children disruptive behavior $(\mathrm{d}=0.27)$, therefore concluding that this program is an effective intervention for child behavior problems. 
In Portugal, the IY has already been translated and implemented (see Webster-Stratton, Gaspar, \& Seabra-Santos, 2012 for a review), demonstrating its transportability to the country. Moreover, results from different studies have shown its efficacy in short and long-term in our country (Azevedo et al., 2013a; Azevedo et al., 2013b; Cabral et al., 2009/2010).

The IY directly targets family risk factors by enhancing positive supportive parenting approaches that promote children's social and persistence competence, emotional regulation, problem-solving and pre-academic skills; and decrease negative behaviors through non-violent discipline methods. Also, it aims to promote parent-child bonding and attachment and to improve parental self-control, depression, anger management, communication, and problem solving skills (Webster-Stratton \& Reid, 2010). Besides diminishing harsh and inconsistent parenting, increasing positive parenting (Hutchings et al., 2007; McMahon, 2006) and helping to reduce and prevent children's behavioral problems, IY has also shown to be effective in improving variables such as maternal depression (Hutchings, Bywater, Williams, Lane, \& Whitaker, 2012). These changes are interpreted mainly as a result of applying problem solving and communication skills (Webster-Stratton \& Hammond, 1999), which may also contribute to reducing conflicts/difficulties in the couple. Moreover, one may speculate that some of the activities included in the Program, such as Caring Days (which aim to strengthen support between partners), or giving praise to other adults (which contributes to stimulate couples interaction and focus parents on the positive characteristics of their partners) may also have an impact on couples dynamics, by improving communication between partners and helping them to focus on positive aspects of the relationship.

The foundation of the program is brief video vignettes of modeled parenting skills shown by a therapist to groups of 8-12 parents. The videos demonstrate social learning and child development principles and serve as stimulus for focused discussions, problem solving, and collaborative learning. The program also relies on performance training methods and group training including role play, practice activities, and live feedback from the therapist and other group members. It also emphasizes a commitment to group members' self-management, thus empowering parents who are often seeking help at times of low self-confidence and intense feelings of guilt and self-blame (Webster-Stratton, 1990). By using group process, the program is not only more cost-effective but also addresses an important risk factor for children with conduct problems: family isolation and stigmatization.

This study expands the literature regarding short and long-term effects of the IY on Portuguese preschooler's mothers by replicating and extending this intervention to Portuguese children with oppositional/aggressive behaviors and analyzing its effectiveness on family outcomes, namely for improving mother-child interactions quality. Moreover, it focuses on a less 
studied topic with the IY Program: variables of the couple's intimacy that may change after the program, as a consequence of the above mentioned characteristics. Therefore, the first aim of this paper is to evaluate the short-term effectiveness of the IY intervention in improving mothers observed positive parenting practices, coaching skills and mothers' self- reported dysfunctional parenting practices by comparing an intervention group (IG) to a waiting-list control group (CG). Furthermore, it is our aim to describe the long-term outcomes in mother-child interactions in the intervention group. In line with previous research (Gardner, Shaw, Dishion, Supplee, \& Burton, 2007), we expect that six-months after the baseline assessments more positive parenting and coaching skills will be observed in mother-child interactions in the IG. It is also expected that mothers in the IG will report less harsh and dysfunctional parenting practices than mothers in the CG.

The second aim is to examine whether there is change on secondary outcome measures, such as mothers' depressive symptoms, mothers' perceived marital quality and mothers' perception of children's behavior as a result of having attended the IY intervention, comparing to mothers in the CG. In addition, it is our aim to examine whether these results, obtained after the IY intervention, are sustained after 12 months follow-up.

\section{Methods}

\section{Participants}

Participants in the research were drawn from an existing sample of 125 families that had been previously randomized to one of two conditions: intervention group (IG, N =69) and waitlist control group ( $C G, N=56$ ). This larger sample was collected for a longitudinal RCT study (from now on referred to as main trial) on the effectiveness of the IY Parents and Teachers Program in Portugal, with preschool children at risk for externalizing behavior disorders and/or behaviors predictive of later $\mathrm{AD} / \mathrm{HD}$ diagnosis. To be eligible for the main trial, children must have fulfilled the following inclusion criteria: (1) young children between 3 and 6 years old; (2) parents' reports of child's behaviors equal to or above the Portuguese borderline cut-off points (Abreu-Lima et al., 2010 ) on the Conduct Scale $(\geq 5)$ or on the Hyperactivity Scale $(\geq 7)$ of the Strengths and Difficulties Questionnaire (SDQ; Goodman 1997); (3) mothers willing to attend an IY group. Children were excluded whenever they had a formal diagnosis of neurological or developmental disorder (e.g., autism), severe developmental delay, or in case they were undergoing pharmacological or psychotherapeutic intervention. In addition, for the present study, other inclusion criteria must have been fulfilled: (1) mother's reports of behavior problems equal or 
above the 85th percentile on at least one of two subscales of the Preschool and Kindergarten Behavior Scales (PKBS-2; Merrell, 2002; Portuguese version by Major, 2011): Antisocial/Aggressive subscale (cut-off point $=14$ ); Oppositional/Explosive subscale (cut-off point = 19) ; (2) having valid recorded mother-child interactions at baseline. After applying these criteria, 83 families were included in this study as follows: forty-four in the IG and thirty-seven in the CG (see flowchart in Figure 1). 
Families with children aged 3-6 years, eligibility criteria for main trial fulfilled (equal or above Portuguese borderline cutoff on SDQ Conduct Scale or Hyperactivity Scale $(n=125)$

Eligibility criteria for present trial fullfilled $(n=83)$

- $\quad$ Above cut-off on PKBS AA ( $\geq 14$ ) and/or PKBS OE ( $\geq 19$ )

- Having valid recorded mother-child interactions at baseline

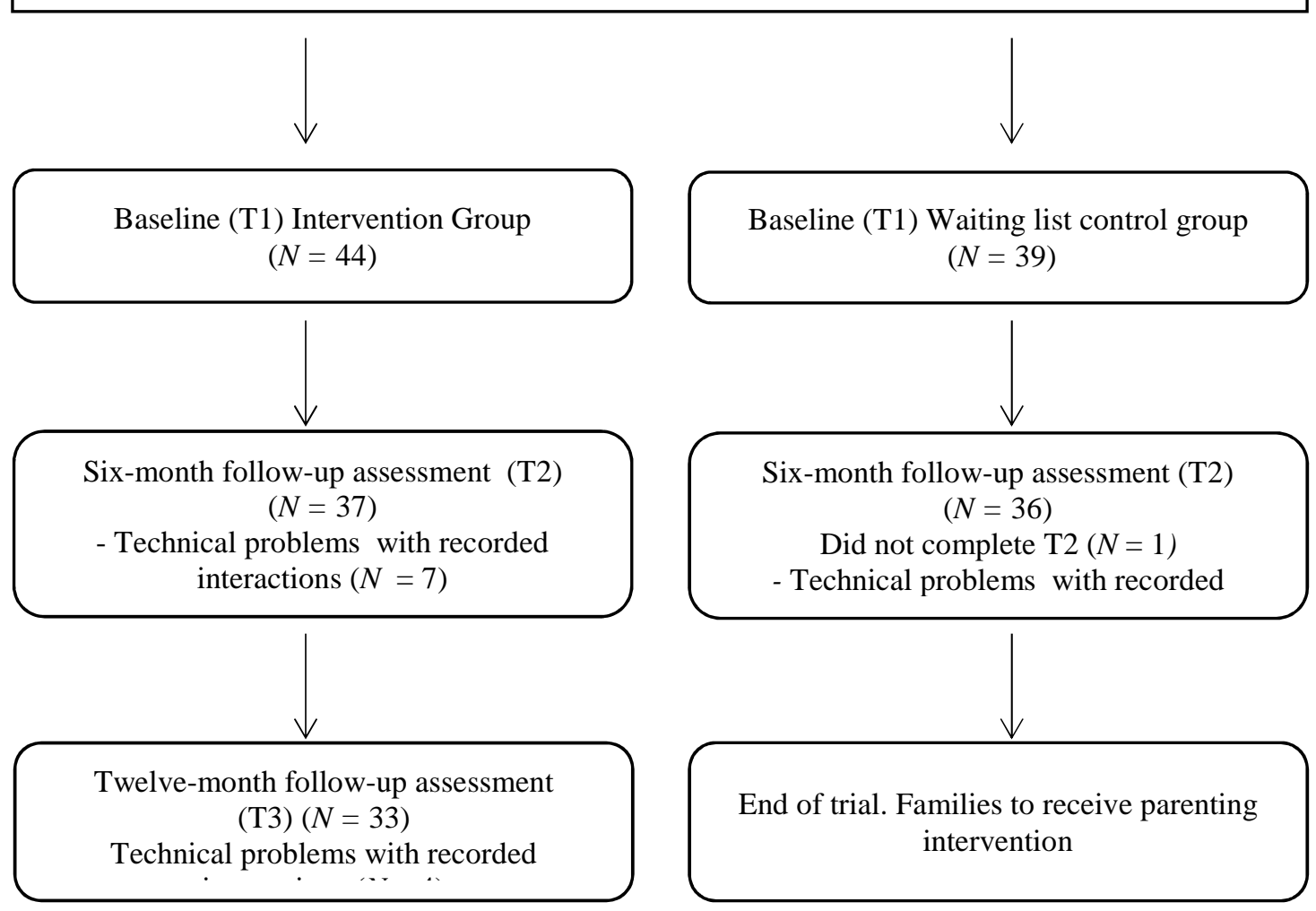

Figure 1. Flowchart of participants through each stage of the trial 
The characteristics of both the intervention group and the control group participants are presented in Table 1. Groups did not significantly differ in socio-demographic characteristics at baseline. Mothers' data are used for the primary analyses because most primary caregivers (94\%) were biological mothers.

Most of the mothers were either married or in a de facto union (82.6\%), with a mean age of 34.47 ( $\mathrm{SD}=4.90$ ) and highly educated (55.4\% had a university degree). Twenty-one percent of the mothers self-reported depression symptoms above the clinical cut-off $(17 ; \mathrm{M}=9.74, \mathrm{SD}=7.75)$ on the Beck Depression Inventory (Beck et al., 1961). Study children included 61 boys and 22 girls, with a mean age of 54.55 months ( $S D=11.53)$. Half of the children were clinically referred (53\%). Regarding oppositional and aggressive symptoms, $97.7 \%$ children in the intervention group ( $\mathrm{M}=$ 22.15; $\mathrm{SD}=2.75)$ and $84.6 \%$ children in the control group $(M=21.30 ; \mathrm{SD}=3.15)$ were at moderate (85th percentile) or high risk level (95th percentile) on the Oppositional/Aggressive PKBS subscale. In the Anti-Social/Aggressive 70\% percent of children in the intervention group $(\mathrm{M}=$ $16.88 ; \mathrm{SD}=6.66)$ and $82 \%$ of children in the control group (M = 16.64; $\mathrm{SD}=5.24)$ were at moderate or high risk level. Moreover, $81.9 \%(\mathrm{~N}=68)$ of the children who met the present paper inclusion criteria also showed signs of co-occurring hyperactive and inattentive behaviors, using as cut-off point parents' reports of $\mathrm{AD} / \mathrm{HD}$ behaviors equal or above the 80th percentile $(\geq 21)$ on the Werry-Weiss-Peters Activity Scale (WWPAS; Routh 1978). These children had previously been included in Azevedo et al. study (2013). 
Table 1. Participants Socio-Demographics Characteristics

\begin{tabular}{|c|c|c|c|c|c|}
\hline \multirow[b]{2}{*}{ Child Variables } & \multicolumn{2}{|l|}{ Group } & \multirow[b]{2}{*}{$\begin{array}{l}\text { Test } \\
\left(t / x^{2}\right)\end{array}$} & \multirow[b]{2}{*}{$\begin{array}{l}\text { Sig } \\
(p)\end{array}$} & \multirow[b]{2}{*}{$\begin{array}{c}\text { Eta } \\
\text { squared } \\
\left(\eta^{2}\right)\end{array}$} \\
\hline & $\begin{array}{c}\text { IG } \\
(n=44)\end{array}$ & CG $(n=39)$ & & & \\
\hline Child's age (months): Mean $\pm S D$ & $53.32 \pm 10.78$ & $55.92 \pm 11.90$ & -1.04 & 0.29 & $0.22 *$ \\
\hline Child's gender (male): no (\%) & $31(70.05 \%)$ & $30(76.9 \%)$ & 0.44 & 0.50 & 0.07 \\
\hline Referral: no (\%) & & & 1.21 & 0.54 & 0.01 \\
\hline Clinic & $13(29.50 \%)$ & $13(33.30 \%)$ & & & \\
\hline Community & $13(29.50) \%$ & $21(53.80 \%)$ & & & \\
\hline \multicolumn{6}{|l|}{ SDQ Cut-Off Point: Mean $\pm S D$} \\
\hline $\mathrm{CP}(\geq 5)$ & $6.27 \pm 1.84$ & $5.92 \pm 1.45$ & 0.94 & 0.34 & $0.21^{*}$ \\
\hline \multicolumn{6}{|l|}{ PKBS Cut-Off Point: Mean $\pm S D$} \\
\hline $\mathrm{OE}(\geq 19)$ & $22.15 \pm 2.75$ & $21.30 \pm 3.15$ & 1.31 & 0.19 & $0.28^{*}$ \\
\hline $\mathrm{AA}(\geq 14)$ & $16.88 \pm 6.66$ & $16.64 \pm 5.24$ & 0.18 & 0.85 & $0.04^{*}$ \\
\hline Number of siblings: no (\%) & & & & 0.31 & 0.14 \\
\hline None & $20(45.5 \%)$ & $20(51.3 \%)$ & & & \\
\hline One & $19(43.2 \%)$ & $19(48.7 \%)$ & & & \\
\hline More than one & $5(11.4 \%)$ & - & & & \\
\hline \multicolumn{6}{|l|}{ Families' Variables } \\
\hline Mothers' age (years): Mean $\pm S D$ & $35.38 \pm 4.70$ & $33.49 \pm 4.98$ & 1.76 & 0.08 & $0.39 *$ \\
\hline Mothers' years of education: Mean $\pm S D$ & $14.36 \pm 3.87$ & $12.87 \pm 3.42$ & 1.84 & 0.06 & $0.40^{*}$ \\
\hline Mothers' marital Status: no (\%) & & & & 0.07 & 0.21 \\
\hline Married or living as married & $40(91 \%)$ & $20(74.4 \%)$ & & & \\
\hline Single mother & $2(4.50 \%)$ & $2(5.10 \%)$ & & & \\
\hline Divorced/separated & $2(4.50 \%)$ & $8(20.50 \%)$ & & & \\
\hline Family SES*:no(\%) & & & & 0.06 & 0.20 \\
\hline Low & $13(29.50 \%)$ & $13(33.30 \%)$ & & & \\
\hline Medium & $13(29.50) \%$ & $21(53.80 \%)$ & & & \\
\hline High & $18(40.90 \%)$ & $5(12.80 \%)$ & & & \\
\hline Mother's depressive symptoms (BDI): Mean $\pm S D$ & $9.00 \pm 7.24$ & $10.15 \pm 8.67$ & 0.41 & 0.67 & $0.14^{*}$ \\
\hline
\end{tabular}

Notes: $S D=$ Standard deviation; SES=Socioeconomic Status - defined using a standardized classification developed for the Portuguese population considering three categories (Almeida 1988): low (e.g., unskilled workers; industry, transport, agriculture workers); medium (e.g., intermediate technicians; administrative); and high (e.g., owners and entrepreneurs, managers, scientific and intellectual professionals). Based on this classification the family's SES was defined taking on the basis of the highest professional category and educational level of both parents; * Cohen's $d$ 


\section{Procedures}

The procedures used during the different phases of this study were the same as those used in the main trial: families were recruited in two cities, Coimbra and Porto, from January 2009 to September 2011 from local clinics, child mental health and developmental services, pediatricians' offices, community and preschools (using fliers), and a newspaper advertisement was published. Before the baseline assessment, written informed consent was obtained from the participating families and the main trial was approved by the hospitals' Research Ethics Committees and by the National Commission of Data Protection.

The 125 parents of the main trial eligible children were invited to participate in the study and were subsequently assessed (baseline assessment) by two independent trained psychologists, before the intervention took place. Assessments were conducted in a university community service and at a central hospital and took place at three moments: time 1, preintervention (baseline, T1); time 2, post-intervention (six months after baseline, T2); time 3, follow-up (12 months after baseline, T3). At baseline, the assessment protocol for all of the families in the main trial comprised a semi-structured interview, parent-report measures, a laboratory-based mother-child interaction observation, and psychological testing of the child. Two questionnaires (SDQ and the PKBS-2) were also sent to the child's pre-school teacher (Seabra-Santos et al., 2013). After T1, the children were stratified by age and gender, and randomly allocated to a IG or a CG. The assessment protocol was repeated for both groups six months after baseline. The intervention group was further assessed 12 months after baseline. Although all efforts were made to keep the evaluators blind to the families study condition in the post-intervention assessment, this was no longer possible at the 12-month follow-up assessment as only intervention families were evaluated because, for ethical reasons, IY was offered to the CG families after time 2 assessment.

\section{Measures}

Given the aims of the present study and the characteristics of the sample, a set of specific measures was selected. These are described below. All measures completed by parents and teachers were available in Portuguese (see Seabra-Santos et al., 2013 for the description of the entire protocol and for previous studies with the selected measures with Portuguese samples).

Screening measures: Mother Reports of Children Behavior

The SDQ (Goodman, 1997) is a 25-item inventory designed as a brief behavioral screening measure to assess the occurrence of particular behaviors that have been associated with conduct 
problems: hyperactivity, emotional symptoms, peer problems and pro-social behavior in children. It has been translated and adapted for use in Portugal (Fleitlich et al., 2005). The level of internal consistency as assessed by Cronbach alpha for the subscales used with this sample was low 0.46 and 0.57 respectively); nevertheless the SDQ has been included in similar studies (e.g., Hutchings et al. 2007) and has demonstrated good psychometric properties with English samples (Goodman, 2001) and acceptable ones with other Portuguese samples (e.g., a $\alpha=0.60$ for hyperactivity and a $\alpha=0.59$ for conduct subscale in the Abreu-Lima et al. [2010] study). In this study the SDQ was only used as a first screening measure, to be confirmed by other inclusion criteria (measured by the PKBS-2 subscales).

\section{Ratings of Mother-Child Interaction Behaviors: Observation}

The Dyadic Parent-child Interaction Coding System (DPICS; Robinson \& Eyberg, 1981) is an observational measure used to assess the quality of parent-child interaction. It consists of separate behavior categories covering parent and child behaviors which are coded as present or absent for each 5-min segment, during a 25- minute period. Following previous Portuguese and international research (Gaspar \& Alarcão, personal communication, February 4, 2010; Hutchings et al. 2007), the current study used two composite categories for the observed parenting: (1) Positive Parenting (labelled and unlabeled praise, positive affect, physically positive behavior and problem-solving); and (2) Coaching (descriptive/encouragement statements and questions, reflective statements and questions). Two trained independent observers who were blind to the hypotheses of the study and the families' group status coded the valid interactions and an overall mean of $76 \%$ inter-rater agreement was achieved. In this sample, intra-class correlations for the variables analyzed were 0.97 and 0.91 for mothers Positive Parenting and Coaching scores respectively.

\section{Mother Reports of Parenting Practices, Depressive Symptoms and Couple's Intimacy}

The Parenting Scale (Arnold et al., 1993) is a thirty-item inventory measuring dysfunctional discipline practices through three different subscales: Laxness (11 items), Overreactivity (10 items) and Verbosity (7 items). Internal consistency in the original study was adequate, ranging from 0.63 to 0.84 (Arnold et al., 1993). In Portuguese exploratory non-clinical samples with preschool-age children (see Seabra-Santos et al., 2013 for these studies) the PS has shown lower levels of internal consistency (from 0.63 to 0.74 ), and even lower for the Verbosity subscale (0.41).The same was found for this study: Cronbach alpha coefficients for this scale range 
between 0.51 (Verbosity) to 0.65 (Overreactivity), 0.73 (Laxness) and 0.76 (Total score); The Beck Depression Inventory (BDI; Beck et al., 1961) is a twenty-one item self-report inventory measuring the severity of symptoms associated to depression. Respondents rate the intensity of 21 depressive symptoms on a 0 (no symptomatology) to 3 (severe symptomatology) scale, and a score is derived from summing these ratings. The BDI was translated and adapted to Portuguese population by Vaz Serra and Pio Abreu (1973) and has demonstrated good psychometric properties. In our sample internal consistency was 0.88. The Personal Assessment of Intimacy in Relationships (PAIR, Shaefer \& Olson, 1981) is a 36-item questionnaire which assesses marital quality. More specifically, it assesses the degree of intimacy in a dyadic relationship. Higher scores in its different subscales are related to a more positive perception of the couple's relationship. The PAIR was translated and adapted to Portuguese population by Moreira, Amaral and Canavarro (2009) and has demonstrated good psychometric properties (Cronbach alpha coefficients between 0.71 and 0.88 ). The Portuguese version comprises three factors: Personal Validation (14 items), Communication (10 items) and Openness to Exterior (5 items) with Cronbach alpha coefficients on our sample of $.79, .86$ and .88 respectively.

\section{Mother Reports of Children Behavior Outcomes}

The Preschool and Kindergarten Behavior Scales-2nd Edition (PKBS-2, Merrell, 2002) is an 80 -item behavior rating scale designed to measure social skills and problem behaviors of preschoolers. This instrument can be completed by parents, teachers or other caregivers and comprises two separate scales: a 34-item Social Skills Scale and a 46-item Problem Behavior Scale. For the aim of the present paper we analyzed Oppositional/Explosive (O/E) (9 items; score range from 0 to 27) and Antisocial/Aggressive (A/A) (12 items; score range from 0 to 36) externalizing behavior problems subscales. The PKBS-2 was translated and adapted to Portuguese children by Major (2011) and has demonstrated good psychometric properties (Cronbach alpha coefficients between 0.76 and 0.97). In the present sample, Cronbach alpha coefficients for the subscales used were 0.83 and 0.60 for the A/A and $0 / E$ subscales, respectively.

\section{Parent's Self-Reported Program Satisfaction: Consumer Satisfaction}

Incredible Years Parent Program Satisfaction Questionnaire (Reid, Webster-Stratton, \& Beauchaine, 2001) is a fifty-three item questionnaire used to measure parental satisfaction at the end of the program. Mothers are asked to rate the program regarding overall satisfaction, the usefulness of the parenting techniques, the difficulty of implementing them, and the group leaders 
skills. Mothers are also asked to comment on their feelings concerning the group and to indicate which aspects of the sessions were the most helpful and which they most liked/disliked.

\section{Intervention Protocol: The Incredible Years Basic Parent Training}

The IY BASIC Parent Program (Webster-Stratton, 2001) main goals are: to strengthen the parent-child relationship and encourage child cooperation; to increase parents' nurturing and positive parenting skills; to encourage effective limit setting; and to promote the use of nonviolent discipline strategies.

The 14-week intervention was delivered weekly to nine groups of nine to twelve parents in two-hour sessions that took place either at a university community service (82\% of groups) or in a central hospital (18\% of groups). All sessions took place in the evening (after work, from 6 to $8 \mathrm{pm}$ ) and partners were strongly encouraged to attend. Supervised childcare was offered to children, as well as a light snack to parents and children, in order to enhance parents' attendance. Like in other studies analysing the effects of IY on children behaviors (e.g., Posthumus, Raaijmakers, Maassen, van Engeland, \& Matthys, 2012) two booster sessions were carried out for clinical and ethical reasons (in order to review parenting strategies, discuss new problems, prevent relapse, and reinforce parents' support). The first one took place nine months after baseline (between assessments T2 and T3); and the second one 15 months after baseline (after assessment T3).

Each session followed the same structure and included a review of the previous one, feedback and discussion about parents' home activities (e.g., skill practicing, buddy-calls) introduction of a new topic (e.g., brainstorming), presentation of video vignettes (with Portuguese subtitles) illustrating parenting skills, group discussion and new strategies practice (e.g., roleplays). In order to promote the relationship between parents and leaders and to keep parents involved in the group training, the group leaders called the parents every week. In addition, individual extra time was offered at the beginning of each session and handouts were sent to parents who missed sessions (Webster-Stratton, 2001).

Training and treatment integrity: Assumptions of fidelity and integrity regarding treatment were respected.The Incredible Years Program has a certification/accreditation process which is about delivering the contents of the program in a collaborative way and aims to maximize fidelity issues. In this trial, the program was delivered by six trained group leaders with child mental health psychology or psychiatry and teaching background and at least 10 year of experience in their fields. All of them had followed the Incredible Years accreditation process to ensure program delivery quality: leaders had attended the accredited three-day IY basic leader 
training by an IY-accredited trainer; had run a pilot group prior to the study; had received videotape feedback on a session by an independent IY trainer who evaluated the treatment implementation fidelity, and had either been accredited as group leaders $(n=4)$ or were undergoing the accreditation process $(n=2)$.

To ensure a minimum integrity level, $80 \%$ of all the sessions were videotaped (124 sessions) for weekly self-evaluation and for regular peer supervision. Group leaders received support and consultation from an IY-accredited trainer. Also, group leaders closely followed the IY protocol; provided standardized materials and translated handouts for all parents; completed leader checklists for $80 \%$ of all delivered session for monitoring protocol adherence; reviewed peer and self-evaluation questionnaires as well as the weekly parent satisfaction questionnaires to check parents' participation and engagement in the program's contents and methods (e.g., use of video, role plays, group discussion).

\section{Program Attendance Rate}

Attendance was high with an average of 11 sessions (out of 14 ) being attended and $81.5 \%$ of mothers attending nine or more group sessions.

\section{Attrition}

Attrition rates concerning assessment completion were generally low. From the 83 families assessed at baseline, 73 (88\%) completed T2 assessment (six months after baseline). From the 10 families who did not complete the T2 assessment, seven were from the IG and two from the CG; another one from the CG dropped out of the trial. No significant differences were found between the two groups regarding the number of cases lost for six-month post-intervention assessment $\left(\chi^{2}=1.31, \mathrm{p}=0.25\right)$.

Regarding the 12-month post-intervention assessment, data were obtained for $75 \%$ of the families in the IG. This means that, from the 44 families who completed T1 assessments, 11 were lost to the T3 assessment.

\section{Post-treatment Therapy and Medication}

From the thirty-three (75\%) treated children assessed at T3, four (7.2\%) had meanwhile been referred for additional treatment (outpatient clinics); and two (3.7\%) had initiated pharmacological intervention. No statistically significant differences emerged on sociodemographic and clinical measures between children referred for additional treatment or 
pharmacological intervention and children who had not started medication or any other additional treatment. Post-treatment therapy/intervention/medication is therefore an implausible explanation for the maintenance of treatment effects.

\section{Data Analysis}

Statistical analyzes using SPSS 20.0 were performed. The inclusion criteria for this study are based on the data provided by primary caregivers (mostly mothers or, in some cases, other female figures) at baseline assessments. However, results from fathers who participated in the trial were also collected and will be explored in future papers.

Baseline comparisons of continuous and categorical variables were done with t- and chisquare tests, respectively. Analysis of variance using the General Linear Model (GLM) for Repeated Measures were performed, more specifically MANOVAs (for multivariate analysis) and ANOVAs (for total scores), with Group as the between-subjects factor and Time as the within-subjects factor. The outcome measures at T2 and T3 were directly compared in order to test the maintenance of effects. Greenhouse-Geisser corrections for violations of sphericity and compound symmetry were used.

Effect sizes (ES) were calculated with eta square ( ${ }^{2}{ }^{2}$ ) and classified according to Cohens' principles: 0.01 for a small effect, 0.06 for a medium effect and 0.14 for a large effect size (Cohen, 1988). The significance level used was .05. An intention-to-treat analysis (ITT) was also carried out, which included all allocated cases and adopted the conservative assumption of no change from baseline to post-intervention in lost cases.

\section{Results}

Group comparisons at baseline

ANOVA's revealed no significant differences between groups at baseline (see Table 2 for $\mathrm{M}$ and SD), except for mothers' DPICS observed coaching variable $(C G<I G ; t(79)=2.69, p=0.00)$. 
Short-term Intervention Effects

These findings are summarized in Tables 2 and 3, where means and standard deviations for both groups at baseline and post-intervention assessment, results of repeated measures MANOVAs (Group X Time effect) and ANOVAs and the Eta Squared are reported. In the text, only outcomes for participants who have completed the assessments will be reported. However, the results from the ITT analysis are also presented in Tables 2 and 3. In general, the two analyses produce identical results.

\section{DPICS: Observed Mother-Child Behaviors}

The overall $F$ in the DPICS observational measure MANOVA was significant $(F(2,71)=$ $10.61, \mathrm{p}=0.00,2 \mathrm{p}^{2}=0.13$ ). Univariate tests indicate that mothers in the IG had a greater increase in positive parenting behaviors while interacting with their children than mothers in the CG (F (2, 71) $=18.91, p=0.00, ? p^{2}=0.21$. Regarding mothers observed coaching skills, scores increased in the IG, while they decreased in the CG, showing a significant group X time interaction effect (F $\left.(2,71)=8.27, \mathrm{p}=0.00, ? \mathrm{p}^{2}=0.10\right)$.

\section{Mother's Self-reported Measures}

As presented in Table 2, the overall $\mathrm{F}$ in the domain of mothers' self-rating parenting practices was significant $\left(F(2,77)=12.45, \mathrm{p}=0.00\right.$, $\left.\mathrm{p}^{2}=0.35\right)$. Univariate tests in this domain also indicate that there was a larger decrease in mothers PS Total scores $(F(1,76)=29.38, p=$ 0.00 , ? $\mathrm{p}^{2}=0.29$ ). Examining the Arnold subscales separately, there was a larger decrease in Laxness $\left(\mathrm{F}(2,77)=4.82, \mathrm{p}=0.03,0 \mathrm{p}^{2}=0.06\right)$, Overeactivity $\left(\mathrm{F}(2,77)=15.54, \mathrm{p}=0.00\right.$, ? $\mathrm{p}^{2}=$ $0.18)$ and Verbosity $\left(\mathrm{F}(2,77)=27.07, \mathrm{p}=0.00\right.$, $\left.2 \mathrm{p}^{2}=0.27\right)$ practices in mothers in the IG when compared to mothers in the CG.

Regarding couple's intimacy, the overall $F$ was not significant $(F(2,57)=2.01, p=0.12$, ? $\mathrm{p}^{2}=0.10$ ). However, univariate tests revealed a significant interaction effect on Openness to Exterior subscale: mothers in the IG reported a greater increase on the $0 / E$ subscale $(F(2,57)=$ $5.34, \mathrm{p}=0.02$, $2 \mathrm{p}^{2}=0.08$ ) from baseline to six-month post-intervention assessment. No other significant group $\mathrm{X}$ time interaction effects were found on PAIR subscales.

No significant interaction effects were found for mother's depressive symptoms from baseline to six-month post-intervention assessments. 


\section{Mother Reports of Children Behavior Outcomes}

Regarding children oppositional behaviors reported by mothers, the overall $\mathrm{F}$ was not significant $\left(F(2,77)=2.19, \mathrm{p}=0.11\right.$, ? $\left.\mathrm{p}^{2}=0.05\right)$. However, univariate tests revealed a significant interaction effect on PKBS Oppositional/Explosive subscale $\left(F(2,77)=4.44, p=0.03\right.$, 国 $\mathrm{p}^{2}=0.05$ ): from baseline to six-month post-intervention assessments, mothers in the IG reported a larger decrease in children's oppositional/aggressive behaviors comparing to the CG. No significant interaction effect was found for anti-social/aggressive behaviors reported by mothers (PKBS$\mathrm{A} / \mathrm{A})$. 
Table 2. Descriptive statistics, univariate and multivariate main and interaction effects

\begin{tabular}{|c|c|c|c|c|c|c|c|c|c|c|}
\hline \multirow{3}{*}{ Variable } & & \multicolumn{7}{|c|}{ Per protocol analysis } & \multicolumn{2}{|c|}{$\begin{array}{l}\text { Intention to treat } \\
\text { analysis } \\
(\mathrm{IG}=44 ; \mathrm{CG}=39)\end{array}$} \\
\hline & \multicolumn{4}{|c|}{ Intervention Group } & \multicolumn{2}{|c|}{ Control Group } & \multirow{3}{*}{$\begin{array}{c}\text { Group X Time } \\
\qquad(F, p)\end{array}$} & \multirow{3}{*}{$\begin{array}{l}\mathrm{ES} \\
\eta_{p}{ }^{2}\end{array}$} & \multirow{3}{*}{$\begin{array}{c}\text { Group X Time } \\
\qquad(F, p)\end{array}$} & \multirow{3}{*}{$\begin{array}{l}\text { ES } \\
\eta_{p}^{2}\end{array}$} \\
\hline & $N$ & Baseline & Post & $N^{a}$ & Baseline & Post & & & & \\
\hline \multicolumn{7}{|c|}{ Lab observed behaviors } & & & & \\
\hline DPICS & 37 & & & & & & $10.61(0.00) * *$ & 0.13 & $10.58(0.00)^{* *}$ & 0.23 \\
\hline Positive Parenting & & $\begin{array}{c}19.64 \pm 12.4 \\
1\end{array}$ & $30.37 \pm 14.09$ & 36 & $15.75 \pm 8.83$ & $14.22 \pm 6.79$ & $18.91(0.00) * *$ & 0.21 & $18.72(0.00)^{* *}$ & 0.20 \\
\hline Coaching & & $\begin{array}{c}23.37 \pm 13.0 \\
6\end{array}$ & $29.00 \pm 15.09$ & 36 & $15.33 . \pm 9.11$ & $12.58 \pm 7.06$ & $8.27(0.00)^{* *}$ & 0.10 & $8.52(0.00) * *$ & 0.10 \\
\hline \multicolumn{11}{|c|}{ Mother self-reports } \\
\hline PS & 42 & & & 37 & & & $12.45(0.00) * *$ & 0.35 & $14.18(0.00)^{* *}$ & 0.36 \\
\hline Total & & $3.64 \pm 0.58$ & $3.01 \pm 0.47$ & & $3.73 \pm 0.62$ & $3.66 \pm 0.58$ & $29.38(0.00) *$ & 0.29 & $34.48(0.00)$ & 0.30 \\
\hline Laxness & & $3.01 \pm 0.80$ & $2.59 \pm 0.75$ & & $3.12 \pm 1.00$ & $3.02 \pm 0.91$ & $4.82(0.03) * *$ & 0.06 & $6.18(0.01) * *$ & 0.07 \\
\hline Overreactivity & & $3.69 \pm 0.77$ & $3.05 \pm 0.68$ & & $3.72 \pm 0.88$ & $3.71 \pm 0.77$ & $15.54(0.00) * *$ & 0.18 & $18.03(0.00) * *$ & 0.19 \\
\hline Verbosity & & $4.27 \pm 1.07$ & $3.28 \pm 0.81$ & & $4.22 \pm 0.81$ & $4.25 \pm 0.78$ & $27.07(0.00) * *$ & 0.27 & $30.66(0.00) * *$ & 0.28 \\
\hline PAIR & 34 & & & 25 & & & $2.01(0.12)$ & 0.10 & $2.06(0.11)$ & 0.09 \\
\hline Total & & $\begin{array}{c}85.09 \pm 20.6 \\
4\end{array}$ & $84.43 \pm 22.24$ & & $\begin{array}{c}94.08 \pm 22.0 \\
8\end{array}$ & $87.28 \pm 18.87$ & $1.65(0.20)$ & 0.02 & $1.34(0.25)$ & 0.02 \\
\hline Op/Ext & & $11.30 \pm 4.05$ & $12.12 \pm 3.56$ & & $12.44 \pm 3.83$ & $11.86 \pm 3.37$ & $5.34(0.02) * *$ & 0.08 & $5.40(0.02) * *$ & 0.08 \\
\hline$S / V$ & & $33.25 \pm 9.58$ & $33.34 \pm 9.77$ & & $35.92 \pm 9.98$ & $33.20 \pm 8.19$ & $2.18(0.14)$ & 0.03 & $1.95(0.16)$ & 0.03 \\
\hline
\end{tabular}


IY in Portuguese Families of Preschoolers with Oppositional/Defiant Symptoms $\mid 20$

\begin{tabular}{|c|c|c|c|c|c|c|c|c|c|c|}
\hline $\mathrm{C}$ & & $25.34 \pm 5.80$ & $23.75 \pm 6.38$ & & $28.04 \pm 6.54$ & $25.36 \pm 5.83$ & $0.65(0.42)$ & 0.01 & $0.38(0.53)$ & 0.00 \\
\hline BDI & 40 & $9.02 \pm 7.29$ & $6.61 \pm 5.66$ & 35 & $9.28 \pm 8.25$ & $7.90 \pm 8.13$ & $0.41(0.52)$ & 0.00 & $0.72(0.39)$ & 0.00 \\
\hline \multicolumn{11}{|c|}{ Mother reports on child behavior } \\
\hline PKBS & 42 & & & 37 & & & $2.19(0.11)$ & 0.05 & $3.01(0.05)$ & 0.07 \\
\hline $\mathrm{A} / \mathrm{A}$ & & $17.16 \pm 6.59$ & $14.21 \pm 7.16$ & & $16.56 \pm 5.41$ & $15.28 \pm 5.78$ & $1.57(0.21)$ & 0.02 & $2.23(0.13)$ & 0.02 \\
\hline $0 / E$ & & $22.09 \pm 2.74$ & $18.69 \pm 4.34$ & & $21.65 \pm 3.14$ & $20.25 \pm 3.55$ & $4.44(0.03) * *$ & 0.05 & $6.09(0.01) * *$ & 0.05 \\
\hline
\end{tabular}

Results are expressed as mean \pm standard deviation; DPICS Dyadic Parent-Child Interaction Coding System; PS Arnold Parenting Scale; BDI Beck

Depression Inventory; PAIR Personal Assessment of Intimacy in Relationships Scale: $O p / E x t$ Openness to Exterior; $S / V$ Self Validation; $C$ Communication; PKBS Preschool and Kindergarten Behavior Scales: $O / E$ Oppositional/Explosive; $A / A$ Antisocial/Aggressive; a $N$ differ due to missing data; 'bignificant baseline difference between groups on this measure; Greenhouse-Geisser epsilons were used to correct $p$ values for violations of sphericity and compound symmetry; Significant follow-up assessment differences between the two condition: ** $p \leq .05, *$ Marginal result $(>0.05$ and $<0.10$ ) 
Maintenance Effect for the Intervention Group (T2-T3)

Although a significant change from T1 to T2 was found in outcome variables other than those presented, only the outcome variables for which significant or marginally significant interaction effects (time X condition) were found from $\mathrm{T} 1$ to $\mathrm{T} 2$ are reported in this analysis, as the absence of a control group at $\mathrm{T} 3$ could bias the interpretation of the findings if done otherwise.

\section{Mother's Reported and Observed Measures}

As shown in Table 3, from T2 to T3 the overall F was significant regarding mother-child

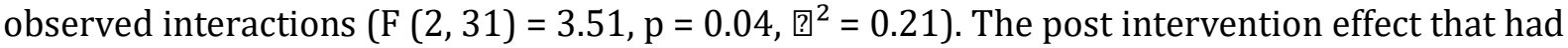
been observed for Coaching (from T1 to T2) faded out by the 12-months follow-up assessment (F $\left.(2,31)=6.21, p=0.01,0^{2}=0.19\right)$. However, regarding Positive Parenting, results remained stable from $\mathrm{T} 2$ to $\mathrm{T} 3$, suggesting the maintenance of post-treatment effects on this specific variable.

Also, regarding mothers' self-rated dysfunctional practices, (Laxness, Overreactivity and Verbosity) from T2 to T3, the overall F was not significant $\left(F(2,33)=0.50, p=0.68\right.$, ? $\left.p^{2}=0.04\right)$ : results on mothers' dysfunctional practices remained stable and non-significant differences were found from $\mathrm{T} 2$ to T3. Overall, the effect sizes ranged from 0.00 to 0.04 , indicating small changes from $\mathrm{T} 2$ to $\mathrm{T} 3$.

On PAIR, no significant change was found from T2 to T3 on the Openness to Exterior variable, suggesting the maintenance of post-treatment effects on that specific variable.

Finally, on mother's perception of children oppositional behavior, results remained stable and non-significant differences were found from $\mathrm{T} 2$ to $\mathrm{T} 3$. 
IY in Portuguese Families of Preschoolers with Oppositional/Defiant Symptoms| 22

Table 3. Differences from post-intervention assessment to follow-up assessment in the intervention condition: Parent and child outcome measures

\begin{tabular}{|c|c|c|c|c|c|c|c|c|c|}
\hline \multirow[b]{2}{*}{ Variable } & \multicolumn{5}{|c|}{ Per protocol analysis } & \multirow{3}{*}{ ES } & \multirow{3}{*}{$N \underline{\mathbf{a}}$} & \multicolumn{2}{|c|}{ ITT } \\
\hline & $N \underline{\mathbf{a}}$ & Baseline & Post & Follow-up & T2-T3 $(F, p)$ & & & $\mathrm{T} 2-\mathrm{T} 3(F, p)$ & ES \\
\hline & & $\mathrm{T} 1$ & $\mathrm{~T} 2$ & $\mathrm{~T} 3$ & & & & & \\
\hline \multicolumn{10}{|c|}{ Lab observed behaviours } \\
\hline DPICS (Mother) & 33 & & & & $3.51(0.04)$ & 0.21 & 44 & $3.94(0.02)$ & 0.19 \\
\hline Positive Parenting & & $18.14 \pm 12.84$ & $31.25 \pm 13.00$ & $28.10 \pm 14.85$ & $1.09(0.30)$ & 0.04 & & $4.07(0.06)$ & 0.11 \\
\hline Coaching $^{1}$ & & $23.81 \pm 15.05$ & $30.69 \pm 14.79$ & $24.00 \pm 15.17$ & $6.21(0.01) * *$ & 0.19 & & $7.31(0.01) * *$ & 0.18 \\
\hline \multicolumn{10}{|c|}{ Mother self-reports } \\
\hline PS & 35 & & & & $0.50(0.68)$ & 0.04 & 44 & $0.25(0.85)$ & 0.01 \\
\hline Total & & $3.69 \pm 0.59$ & $2.99 \pm 0.47$ & $2.97 \pm 0.50$ & $0.07(0.78)$ & 0.00 & & $0.06(0.80)$ & 0.00 \\
\hline Laxness & & $3.03 \pm 0.81$ & $2.55 \pm 0.68$ & $2.44 \pm 0.69$ & $1.62(0.21)$ & 0.04 & & $0.82(0.37)$ & 0.02 \\
\hline Overreactivity & & $3.66 \pm 0.73$ & $3.07 \pm 0.73$ & $3.10 \pm 0.62$ & $0.24(0.62)$ & 0.00 & & $0.04(0.82)$ & 0.00 \\
\hline Verbosity & & $4.29 \pm 1.07$ & $3.26 \pm 0.76$ & $3.31 \pm 0.90$ & $0.19(0.66)$ & 0.00 & & $0.14(0.70)$ & 0.00 \\
\hline PAIR & 35 & & & & & & & & \\
\hline Op/Ext & & $12.04 \pm 3.29$ & $12.50 \pm 3.24$ & $13.04 \pm 3.32$ & $1.08(0.30)$ & 0.04 & & $2.14(0.15)$ & 0.06 \\
\hline \multicolumn{10}{|c|}{ Mother reports on child behaviour } \\
\hline
\end{tabular}

PKBS

35

$\mathrm{O} / \mathrm{E}$

$22.55 \pm 2.38$

$18.55 \pm 4.1$

$18.44 \pm 4.95$

$0.02(0.86)$

0.00

0.75 (0.38)

0.01

Results are expressed as mean \pm standard deviation; DPICS Dyadic Parent-Child Interaction Coding System; PS Arnold Parenting Scale; PAIR Personal Assessment of Intimacy in Relationships Scale: $O p / E x t$ Openness to Exterior; PKBS Preschool and Kindergarten Behavior Scales: $O / E$ Oppositional/Explosive; ${ }^{-}$N differ due to missing data ${ }^{1}$ Significant baseline difference between groups on this measure; ${ }^{*}$ Greenhouse-Geisser epsilons were used to correct $p$ values for violations of sphericity and compound symmetry; Significant follow-up assessment differences between the two condition: ${ }^{* *} p \leq .05,{ }^{*}$ Marginal result $(>0.05$ and $<0.01)$ 
Consumer Satisfaction

After the 14-session program, $98.3 \%$ of the mothers would recommend (3.4\%) or highly recommend (94.9\%) this program to other parents; $98.3 \%$ of the mothers had positive (30.5\%) or very positive feelings about the program (67.8\%); and $84.7 \%$ of the mothers reported that they did not need additional help after the end of the program.

\section{Discussion}

This paper has analysed the short and long-term effects of a parent-based intervention, the IY, on a sample of Portuguese families of preschoolers with ODD behaviors. We compared the baseline (T1) and the six-month post-intervention assessments (T2) of two groups (IG and CG) and it was expected that the changes in mothers observed positive parenting and mothers' selfreported dysfunctional parenting practices, perception of marital quality, depressive symptoms and perceptions of children behavior from T1 to T2 would be greater after the 14-week parent intervention compared to the CG. Moreover, we analyzed whether these post-intervention expected changes were maintained twelve-months after baseline (T3).

Overall, results suggest that the IY intervention can make a positive difference in improving mother-child interactions quality in Portuguese families of preschoolers with ODD behaviors and also in improving children's oppositional behavior. The effects found in the sixmonth post-intervention assessment remained stable over time, as demonstrated by nonsignificant differences and small effect sizes between T2 and T3 assessments. Additionally, significant differences were found on the Openness to Exterior PAIR's subscale which were sustained from T2 to T3. No differences were found between the two groups either on other PAIR subscales or on mothers' depressive scores, from T2 to T3. Finally, mothers in our study were extremely committed to the program, confirming the positive acceptability of IY in Portugal (Azevedo et al., 2013a), as shown by high attendance rates and reported satisfaction.

In summary, these findings are encouraging and add new evidence to support the short and long-term effectiveness of the IY parent program as a successful method of early intervention to promote the development of quality mother-child interaction, some dimensions of quality of marital relationships and children's oppositional behavior in a specific Portuguese sample of families with children with early signs of ODD. Thus, these results extend the demonstrated impact of IY with other subsamples of Portuguese preschool children to a new segment of the Portuguese population, where these programs are still taking their first steps through implementation and research (Webster-Stratton et al. 2012). 
Regarding our major findings (primary outcome), and in line with Gardner, Burton and Klimes (2006) randomized controlled trial, with 76 children referred for conduct problems and follow-ups at six and 18 months, the main improvements found in our study were on mothers observed positive parenting. These results suggest the efficacy of the IY parent program in promoting, in the short and long-term, the development of more positive parenting behaviors in mothers, while interacting with their children. Furthermore, these results are in agreement with mothers' reduction of dysfunctional parenting, such as overreactivity and verbosity practices, although this latest result should be interpreted cautiously due to the low internal consistency of these subscales in our sample. The fact that these changes are sustained after 12 months of followup is extremely relevant, since positive parenting is considered an important mediator of treatment outcomes (Gardner et al., 2006). Moreover, it is recognized that preschoolers with early ODD symptoms may be more sensitive to negative parenting and there is an increased risk of the development of a negative cycle of coercive parent-child interactions and comorbid behavior problems. Therefore, investing in positive parenting behaviors increase is of great preventive value.

Also, in what regards mothers observed coaching behavior, the IY parent program seems to have a short-term effect. This finding is particularly relevant given the differences observed at baseline in coaching behavior between the two groups, with parents in the IG having higher scores on this variable. While this difference would make the potential for higher improvement in the CG, the opposite was observed. Unfortunately, in contrast to the findings of Bywater and colleagues (2009) obtained with 104 parents of preschool children with behavior problems, this postintervention effect faded out by the 12-months follow-up assessment. Some possible explanations can be proposed for this result. Firstly, the intervention program was not successful enough in sustaining this parenting skill. We could speculate that due to the fact that children with ODD symptoms often have self-regulation difficulties, more time would be necessary to allow parents to practice these specific skills (i.e. descriptive persistence and social-emotional comments) more intensively, like in the longer version of IY used by Webster-Stratton, Reid, \& Beauchaine (2011). Secondly, it may also suggest the need for using more methods to reinforce parenting skills and maintain improvements (e.g. additional booster sessions; the establishment of a post-intervention support network for parents).

These results are consistent with and follow the same trend as other Portuguese studies with the IY parenting program. For example, Azevedo and colleagues (Azevedo et al., 2013a; Azevedo et al., 2013b), in the above mentioned study, which aimed to evaluate the efficacy of the IY in reducing hyperactive and inattentive behaviors, also found that the IY intervention improved mothers positive parenting and coaching skills, while it reduced dysfunctional parenting and; 
these results were sustained over time (except for mother coaching behavior results, which also faded out by the 12-months follow-up). Similarly, in a Portuguese community sample of socioeconomically disadvantaged families at risk of developing children behavior problems (Cabral et al., 2009/2010) the IY Parenting Program has also improved parenting practices and increased parents' empathy and sensitivity regarding the child's needs.

In relation to our secondary outcome measures, the IY program results were encouraging. Considering couple's intimacy, although there were no significant differences between intervention and control groups, on what concerns the Communication and Self-Validation subscale from pre- to post-assessments, significant differences were found on the Openness to Exterior subscale and these differences are sustained from T2 to T3. It might be that, as the IY program emphasizes the benefits of having a supportive parents' network, it contributes to increase the couple's willingness to be more open to others, namely, to shared friends. Some possible explanations to the lack of results in the other subscales are: (1) the smaller size of the sample, due to the fact that mothers did not always complete this questionnaire as they considered some of its items too intimate; (2) the fact that among maritally-distressed couples, a simple parenting intervention might not be as effective as interventions that include training in couple-communication (Dadds, Schwartz, \& Sanders, 1987).

Regarding children's behaviors, and in line with the results reported by Menting and colleagues meta-analysis (2013), differences were found between the IY intervention group and the control group in mothers' perceptions of children oppositional behaviors suggesting the efficacy of the IY parent program in reducing oppositional behaviors in the short and long-term. Although different from the results reported in other Portuguese studies in what concerns children oppositional/aggressive problems (Azevedo and colleagues, 2013a); Cabral and colleagues, 2009/10) the improvements in children oppositional behavior found in the present study could be related to the fact that in order to be included in this study, mothers reports of children behavior problems had to be equal or above the 85th percentile on at least one of two subscales of the PKBS-2. The degree of co-morbidity between ODD and AD/HD behaviors is higher than in other studies (e.g. Jones et al., 2007; Webster-Stratton et al., 2011), which can be explained by the low cut-off points considered for inclusion, since we wanted to adress children who might be at-risk for ODD and who were not actually diagnosed. However, we can expect that, as a sample, children in the present study exhibit more oppositional behaviors than those displayed by children in the Azevedo study (2013a), where the main inclusion criteria was the presence of hyperactive behaviors.

Finally, and although many studies have found both high levels of maternal depression and reduction in depression post-intervention (Hutchings et al., 2012) in the present study there 
were low levels of mothers baseline depression scores, which might explain the absence of potential for improvement concerning this variable.

In conclusion, this study offers preliminary support for the short and long term benefits of IY intervention in a sample of Portuguese families with preschoolers presenting early ODD signs. Moreover, it has several strengths. Firstly, it includes a direct-observation measure of motherchild interaction at baseline, six and 12-month assessments, therefore reducing the risk of bias that exists when measures are collected only indirectly, from the participants' point of view (Gardner et al., 2006); secondly, it uses a multi-method assessment, blind evaluators in both assessments and blind participants until group allocation, and a low attrition rate; finally, it is a longitudinal design, including data from a 12-month follow-up. All these positive aspects assure the study interest and validity.

Nevertheless, some weaknesses recommending caution in the interpretation of some of the results should be considered. Firstly, the generalization of findings must be carefully read, due to: a potential sample selection bias, since not all families might have been willing to participate in this study because of its length (this issue must be systematically addressed and evaluated in future studies); the fact that half of the mothers in this sample had attended higher education; and also the context in which this study took place (primarily, a university-based context). Secondly, there was no untreated control group at the 12-month follow-up, nor were evaluators blind to the families' treatment group at this point. By only analyzing variables showing marginal or significant intervention effects from pre- to post-assessment, when compared to control group, we tried to overcome this limitation, but it is impossible to rule out the possibility that the effects found from T1 to T3 may have been caused by maturation or other changes in these families' lives (Webster-Stratton et al., 2012). Thirdly, the small sample size limits the power of findings and the possibility to generalize and reduces the power of the analysis to detect small effects. Finally, due to the lack of reliable Portuguese instruments for this age group, measures were selected based on other similar international studies (e.g., Jones et al., 2007; Hutchings et al., 2007) and on previous data from Portuguese samples (see Seabra-Santos et al., 2013, for revision of studies). Nevertheless, the psychometric properties of some of the measures are motives for concern (e.g., SDQ and Parenting Scale) and must be addressed in future studies.

Overall, results from this study are promising for the efficacy of this intervention in improving quality in parent-child interactions, increasing couples' intimacy on what concerns their willingness to open to the exterior, and improving child behavior. Moreover, mothers' engagement in the intervention was high, with high rates of attendance and low drop-out rates. The findings reported here support the importance of early childhood intervention and the utility of evidence based parenting programs in improving the quality of parent-child relationships and 
families overall quality of life. However, it is important to continue to evaluate the long-term effectiveness of IY and to conduct studies to determine if a larger dose of the program or the combination with other programs, targeting school, the couple or the child more directly, might add clinically significant improvements (Webster-Stratton et al., 2012).

In conclusion, although interventions for at-risk preschool children have been shown to be essential in the quality of parent-child relationships promotion (Hutchings et al., 2007), this kind of procedure is not common in Portugal. Nevertheless, this and other studies have proven that intervening as early as possible can have lifelong benefits and help children with behavioral difficulties and their families to improve their relationships and overall well-being and quality of life.

\section{Acknowledgments}

This work was supported by the Foundation for Science and Technology (PhD grant: SFRH/BD/43562/2008; project research grant: PTDC/PSI-PED/102556/2008, also funded by the program Compete in its component Feder).We are very grateful to all the parents and children that kindly participated in this study; and to our project team (group leaders, evaluators and advisers) for their involvement and constant support.

Declaration of Conflicting Interests

The authors declare that they have no conflict of interests with respect to the present paper research.

\section{References}


Abreu-Lima, I., Alarcão, M., Almeida, A., Brandão, T., Cruz, O., Gaspar, M., \& Santos, M. (2010). Avaliação de intervenções de educação parental: Relatório [Assessment of parental education interventions: Report]. Retrieved from http://www.cnpcjr.pt/preview_documentos.asp?r=3493\&m=PDF

Almeida, L. (1998). O raciocínio diferencial dos jovens portugueses: Avaliação, desenvolvimento e diferenciação [Differential reasoning in Portuguese youth: Assessment, development and differentiation]. Porto: Instituto Nacional de Investigação Científica.

American Psychiatric Association (2000). Diagnostic and statistical manual of mental disorders (4th ed., text rev.). Washington, DC: Author.

Arnold, D., O'Leary, S., Wolff, L., \& Acker, M. (1993). The Parenting Scale: A measure of dysfunctional parenting in discipline situations. Psychological Assessment, 5, 137-144.

Azevedo, A., Seabra-Santos, M. J., Gaspar, M. F., \& Homem, T. (2013a). The Incredible Years Basic Parent Training for Portuguese preschoolers with AD/HD behaviors: Does it make a difference? Child and Youth Care Forum. doi: 10.1007/s10566-013-9207-0

Azevedo, A., Seabra-Santos, M. J., Gaspar, M. F., \& Homem, T. (2013b). A parent-based intervention program involving preschoolers with $\mathrm{AD} / \mathrm{HD}$ behaviors: Are children's and mothers'effects sustained over time? European Child and Adolescent Psychiatry. doi: $10.1007 / \mathrm{s} 00787-013-0470-2$

Baker, B. \&, Heller, T. (1996). Preschool children with externalizing behaviors: Experience of fathers and mothers. Journal of Abnormal Child Psychology. 24, 513-32.

Beck, A., Ward, C., Mendelson, M., Mock, J., \& Erbaugh, G. (1961). An inventory for measuring depression. Archives of General Psychiatry, 4, 53-63.

Belsky, J. (1984). The determinants of parenting: A process model. Child Development, 55, 83-96.

Bloomquist, M., \& Schnell, S. (2002). Helping children with aggression and conduct problems: Best practices for intervention. London: Guilford Press.

Bywater, T., Hutchings, J., Daley, D., Whitaker, C., Yeo, S., Jones, K., ... Edwards, R.T. (2009). Long-term effectiveness of a parenting intervention in Sure Start services in Wales for children at risk of developing conduct disorder. British Journal of Psychiatry, 195, 318-324. doi:10.1192/bjp.bp.108.056531

Cabral, A., Ferreira, A., Santos, J., Gaspar, M., Silva, P., \& Eufrazia, R. (2009/2010). Uma aventura no mundo da família [An adventure in the world of the family]. Gouveia: Edições GAF.

Campbell, S. (1995). Behavior problems in preschool children: A review of recent research. Journal of Child Psychology and Psychiatry, 36, 113-149. doi: 10.1111/j.14697610.1995.tb01657.x 
Campbell, S., Shaw, D., \& Gilliom, M. (2000). Externalizing behavior problems: Toddlers and preschoolers at risk for later maladjustment. Development and Psychopathology, 12, 467-488. doi: $10.1017 /$ S0954579400003114

Cohen, J. (1988). Statistical power analysis for the behavioral sciences (2nd ed.). Hillsdale, NJ: Laurence Erlbaum.

Cummings, E., Davies, P, \& Campbell, S. (2000). Developmental psychopathology and family process. New York: Guilford Press.

Dadds, M., Schwartz, S., \& Sanders, M. (1987). Marital discord and treatment outcome in behavioral treatment of child conduct disorders. Journal of Consulting and Clinical Psychology, 55, 396-403. doi: 10.1037/0022-006X.55.3.396

Fleitlich, B., Loureiro, M., Fonseca, A., \& Gaspar, F. (2005). Questionário de capacidades e dificuldades (SDQ-Por) [Strenghts and Difficulties Questionnaire, Portuguese Version]. Retrieved from www.sdqinfo.org.

Fossum, S., Handegard, B., Martinussen, M., Morch, W. (2008). Psychosocial interventions for disruptive and aggressive behaviour in children and adolescents: A meta-analysis. European Child and Adolescent Psychiatry 17, 438-51.

Gaspar, M., \& Seabra-Santos, M. (2008). First results from the translation, implementation and researching of the Webster-Stratton "Incredible Years" Basic Parenting Programme in Portugal. In C. Canali, T. Vecchiato \& J. Whittaker (Eds.). Assessing the "evidence-base" of intervention for vulnerable children and their families (pp.115-117). Padova: Fondazione Emanuela Zancan onlus.

Gardner, F. (1994). The quality of joint activity between mothers and their children with conduct problems. Journal of Child Psychology \& Psychiatry, 35, 935-949. doi: 10.1111/j.14697610.1994.tb02303.x

Gardner, F., Burton, J., \& Klimes, I. (2006). Randomised controlled trial of a parenting intervention in the voluntary sector for reducing child conduct problems: Outcomes and mechanisms of change. Journal of Child Psychology and Psychiatry, 47, 1123-1132. doi: 10.1111/j.1469-7610.2006.01668.x

Gardner, F., Shaw, D., Dishion, T., Supplee, L., \& Burton, J. (2007). Randomized prevention trial for early conduct problems: Effects on proactive parenting and links to toddler disruptive behavior. Journal of Family Psychology, 21, 398-406. doi: 10.1037/0893-3200.21.3.398

Goodman, R. (1997). The Strengths and Difficulties Questionnaire: A research note.Journal of Child Psychology, Psychiatry and Allied Disciplines, 38, 581-586. doi: 10.1111/j.14697610.1997.tb01545.x 
Goodman, R. (2001). Psychometric properties of the strengths and difficulties questionnaire (SDQ). Journal of American Academy of Child and Adolescent Psychiatry, 40, 13371345. doi:10.1097/00004583-200111000-00015

Gomez, R., \& Sanson, A. (1994). Mother-child interactions and noncompliance in hyperactive boys with and without conduct problems. Journal of child psychology and psychiatry, and allied disciplines 35, 477-490. doi: 10.1111/j.1469-7610.1994.tb01735.x

Hutchings, J., Bywater, T., Daley, D., Gardner, F., Whitaker, C., \& Edwards, R. (2007a). Parenting intervention in Sure Start services for children at risk of developing conduct disorder: Pragmatic randomized controlled trial. British Medical Journal, 334, 678-682. doi:10.1136/bmj.39126.620799.55

Hutchings, J., Bywater, T., Williams, M., Lane, E., \& Whitaker, C. (2012). Improvements in maternal depression as a mediator of child behaviour change. Psychology, 3, 795-801. doi: 10.4236/psych.2012.329120

Jones, K., Daley, D., Hutchings, J., Bywater, T., \& Eames, C. (2007). Efficacy of the Incredible Years Basic parent training program as an early intervention for children with conduct problems and ADHD. Child: Care, Health and Development, 33, 749-756. doi:10.1111/j.13652214.2007.00747.x

Katz, L. F., \& Gottman, J. M. (1994). Marital conflict styles and children's emotions. In R. Parke \& S. Kellam (Eds.), Exploring family relationships with other social contexts (pp. 49-74). Hillsdale, N.J.: Lawrence Erlbaum Press.

Linville, D., Chronister, K., Dishion, T., Todahl, J., Miller, J., Shaw, D., Gardner, F. and Wilson, M. (2010). A longitudinal analysis of parenting practices, couple satisfaction, and child behavior problems. Journal of Marital and Family Therapy, 36, 244-255. doi: 10.1111/j.17520606.2009.00168.x

Major, S. (2011). Avaliação de aptidões sociais e problemas de comportamento em idade préescolar: Retrato das crianças portuguesas [Assessment of social skills and behavior problems in preschool ages: Portrait of Portuguese children] (Doctoral Dissertation). Retrieved from https://estudogeral.sib.uc.pt/bitstream/10316/17774/5/Tese_Sofia\%20Major.pdf

Matos, M., Bauermeister, J., \& Bernal, G. (2009). Parent-child interaction therapy for Puerto Rican preschool children with ADHD and behavior problems: A pilot efficacy study. Family Process, 48, 232-252. doi:10.1111/j.1545-5300.2009.01279.x

McGilloway, S., Mhaille, G., Bywater, T., Furlong, M., Leckey, Y., Kelly, P., Comiskey, C., \& Donnelly, M. (2012). A parenting intervention for childhood behavioral problems: A randomized controlled trial in disadvantaged community-based settings. Journal of Consulting and Clinical Psychology, 80, 116127. doi: 10.1037/a0026304 
McMahon, R. (2006). Parent training interventions for preschool-age children. In R. Tremblay, R. Barr \& R. Peters (Eds.), Encyclopedia on early childhood development (May 2006 ed.). Retrieved from http://www.excellenceearlychildhood.com/documents/McMahonRJANGxp.pdf

Menting, A., Orobio de Castro, B., \& Matthys, W. (2013). Effectiveness of the Incredible Years parent training to modify disruptive and prosocial child behavior: A meta-analytic review. Clinical Psychology Review, 33, 901-913. Retirado de http://dx.doi.org/10.1016/j.cpr.2013.07.006

Merrell, K. (2002). Preschool and Kindergarten Behavior Scales (2nd ed.). Austin, TX: PROED.

Moreira, H., Amaral, A., \& Canavarro, M. C. (2009). Adaptação do Personal Assessment of Intimacy in Relationships Scale (PAIR) para a população Portuguesa: Estudo das suas características psicométricas [Adaptation of the Personal Assessment of Intimacy in Realtionships Scale (PAIR) for Portuguese population: Study of its psychometric characteristics]. Psychologica, 50, 353-373.

Patterson, G. (2002). The early development of coercive family process. In J. B. Reid, G. R. Patterson \& J. Snyder (Eds.), Antisocial behavior in children and adolescents: A developmental analysis and model for intervention (pp. 25-44). Washington, DC: American Psychological Association.

Patterson, G., \& Sanson, A. (1999). The association of behavioural adjustment to temperament, parenting and family characteristics among five-year-old children. Social Development, 8, 293-309.doi: 10.1111/1467-9507.00097

Patterson, G., DeGarmo, D., \& Forgatch, M. (2004). Systematic changes in families following prevention trials. Journal of Abnormal Child Psychology, 32, 621-633. doi: 10.1037/a0016732

Perrin, E., Sheldrick, R. McMenamy, J., Henson, B., \& Carter, A. (2013). Improving parenting skills for families of young children in pediatric settings: A randomized clinical trial. JAMA Pediatrics, 4. doi:10.1001/jamapediatrics.2013.2919

Podolski, C., \& Nigg, J. (2001). Parent stress and coping in relation to child ADHD severity and associated child disruptive behavior problems. Journal of Clinical Child Psychology, 30, 501511.

Posthumus, J., Raaijmakers, M., Maassen, G., van Engeland, H., \& Matthys, M. (2012). Sustained effects of Incredible Years as a preventive intervention in preschool children with conduct problems. Journal of Abnormal Child Psychology, 40, 487-500. doi: 10.1007/s10802-011$9580-9$

Reid, J., Webster-Stratton, C., \& Beauchaine, T. (2001). Parent training in Headstart: A comparison of program response among African American, Asian American, Caucasian and Hispanic mothers. Prevention Science, 4, 209-227. doi: 10.1023/A:1013618309070 
Robinson, E., \& Eyberg, S. (1981). The dyadic parent-child interaction coding system: Standardization and validation. Journal of Consulting and Clinical Psychology, 49, 245-250. doi: 10.1037/0022-006X.49.2.245

Rijo, D., Motta, C., Silva, D., \& Brazão, N. (2013, November). A prevalência das perturbações mentais em jovens agressores intervencionados pelo Sistema de Justiça Juvenil Português [The prevalence of mental disorders in young offenders in the Portuguese juvenile justice system]. Paper presented at Mental Health and Juvenile Justice Seminar Lisbon.

Rockhill, C., Collett, B., McClellan, J., \& Speltz, M. (2006). Oppositional Defiant Disorder. In J. Luby (Ed.), Handbook of preschool mental health: Development, disorders and treatment (pp. 80115). New York: Guilford Press.

Sameroff, A. (2009). The transactional model. In A. Sameroff (Ed.), The transactional model of development:How children and contexts shape each other (pp. 3-21). Washington: American Psychological Association.

Sanders, M., Markie-Dadds, C., Tully, L., Bor, W. (2000). The Triple P-Positive Parenting Program: A comparison of enhanced, standard, and self-directed behavioral family intervention for parents of children with early onset conduct problems. Journal of Consulting and Clinical Psychology, 68, 624-640. doi: 10.1037/0022-006X.68.4.624

Sawyer, M., Baghurst, P., Arney, F., Clark, J., Graetz, B., Waites, L. (...), Rey, J., \& Zubrick, S.. (2005). The Mental health of young people in Australia: Child and Adolescent Component of the National Survey of Mental Health and Well-Being. Canberra: Australian Data Archive, The Australian National University.

Schaefer, M., \& Olson, D. (1981). Assessing intimacy: The PAIR Inventory. Journal of Marital and Family Therapy, 7, 47-60.

Seabra-Santos, M. J., Gaspar, M. F., Azevedo, A., Homem, T. C., Leitão, S., Pimentel, M., \& Major, S. (2013). Protocolo de avaliação no âmbito do Projeto "Prevenção/intervenção precoces em distúrbios de comportamento: Eficácia de programas parentais e escolares (PTDC/PSIPED/102556/2008)"-Versão de Investigação. [Protocol of measures for the evaluation of the "Early prevention/intervention in disruptive behavior disorders: Efficacy of parents and teachers programs' Project - Research version]. Retrieved from http://fpce.uc.pt/anosincriveis/protocolo.doc

Shaw, D., Keenan, K., \& Vondra, J. (1994). Developmental precursors of externalizing behavior: Ages 1 to 3. Developmental Psychology, 30, 355-364. doi: 10.1037/0012-1649.30.3.355

Shaw, D., Owens, E., Giovannelli, J., \& Winslow, E. (2001). Infant and toddler pathways leading to early externalizing disorders. Journal of the American Academy of Child and Adolescent Psychiatry, 40, 36-43. 
Vaz-Serra, A., \& Abreu, J. (1973). Aferição dos quadros clínicos depressivos I: Ensaio de aplicação do 'Inventário Depressivo de Beck' a uma amostra portuguesa de doentes deprimidos [Evaluation of clinical depression I: Preliminary use of the 'Beck Depression Inventory' with a Portuguese sample of depressed patients]. Coimbra Médica, 20, 623-644.

Wakschlag, L., Briggs-Goawan, M., Carter, A., Hill, C., Danis, B., Keenan, K., ... Leventhal, B. (2007). A developmental framework for distinguishing disruptive behavior from normative misbehavior in preschool children. Journal of Child Psychology and Psychiatry, 48, 976-987. doi:10.1111/j.1469-7610.2007.01786

Webster-Stratton, C. (1990). Enhancing the effectiveness of self-administered videotape parent training for families with conduct-problem children. Journal of Abnormal Child Psychology, 18, 479-492.Retirado de http://67.199.123.90/library/items/enhancing-the-effectivenessvideotraining_1990.pdf

Webster-Stratton, C. (2001). The parent and child series: A comprehensive course divided into four programs - Leaders' guide. Seattle: The Incredible Years.

Webster-Stratton, C. (2011). The Incredible Years parents, teachers, and children training series: Program content, methods, research and dissemination, 1980-2011. Seattle: The Incredible Years.

Webster-Stratton, C., Gaspar, M., \& Seabra-Santos, M. J. (2012). Incredible Years parent, teachers and children's series: Transportability to Portugal of early intervention programs for preventing conduct problems and promoting social and emotional competence. Psychosocial Intervention (Intervención Psicosocial), 21, 157-169. doi:10.5093/in2012a15

Webster-Stratton, C., \& Hammond, M (1999). Marital Conflict Management Skills, Parenting Style, and Early-onset Conduct Problems: Processes and Pathways. Journal of Child Psychology and Psychiatry, 40, 917-927.

Webster-Stratton, C., \& Reid, M. J. (2010). The Incredible Years parents, teachers, and children training series: A multifaceted treatment approach for young children with conduct problems. In J. Weisz \& A. Kazdin (Eds.), Evidence-based psychotherapies for children and adolescents (2nd ed., pp. 194-210). New York: Guilford Publications.

Webster-Stratton C., Reid J., \& Beauchaine, T. (2011). Combining parent and child training for young children with ADHD. Journal of Clinical Child and Adolescent Psychology, 40, 191-203. doi:10.1080/15374416.2011.546044 
IY in Portuguese Families of Preschoolers with Oppositional/Defiant Symptoms| 34 